Aus dem Anatomischen Institut der Med. Fakultät Okayama (Vorstand: Prof. M. SEKI).

\title{
Histologische Untersuchung des Musculus omohyoideus des Menschen.
}

\author{
人の肩非舌骨筋の組織學的研觉. \\ Von
}

\begin{abstract}
Minoru HANDA. 牛时 稔.
\end{abstract}
(Eingegangen am 15. Februar 1950.)

Trotzdem der M. omohyoideus des Menschen ein dünner Muskel

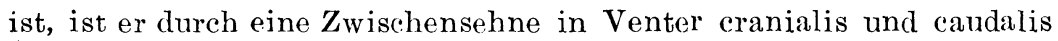
geteilt, welche Tatsache mich interessiert und veranlaßt hat, eingehend zu untersuchen, in welchen Verhältnissen die Dicke der Muskelfasern, ihr Variationskoeffizient und die Gesamtzahl der Muskelfasern zu dem Beruf, der Krankheit, dem Ernährungszustand und vor allem dem Alter und Geschlecht stehen.

Über diesen Muskel gibt es fast keinen histologischen Bericht. Nur teilte KOHASHI (1936) ganz kurz mit, daß der Venter caudalis etwas dickere Muskelfasern führt als der Venter cranialis.

\section{Material und Methode.}

Tabelle 1 .

\begin{tabular}{|c|c|c|c|c|}
\hline & $\begin{array}{c}\text { Körper- } \\
\text { gewicht } \\
(k g)\end{array}$ & $\begin{array}{c}\text { Körper- } \\
\text { größe } \\
\text { (cmi) }\end{array}$ & Todesursache & Beruf \\
\hline $17 \mathrm{~J} . \hat{o}$ & 32.8 & 156 & Lungentuberkulose & Kramladendiener \\
\hline $29 \mathrm{~J} . \quad \hat{0}$ & 60.1 & 154 & Selbstmord & Baue $r$ \\
\hline $69 \mathrm{~J} . \quad \hat{0}$ & 53.2 & 155 & Altersschwäche & Schauspieler \\
\hline $33 \mathrm{~J}$. 우 & 48.2 & 152 & $?$ & $?$ \\
\hline $45 \mathrm{~J}$. 우 & 58.5 & 144 & Akute Herzlähmung & Obdachlose ohne Beruf \\
\hline
\end{tabular}

Diese fünf Leichen wurden, nachdem man in die Arteria femoralis 20\%ige Formalinlösung eingespritzt hatte, einige Monate lang ebenfalls in 10\% ige Formalinlösung eingelegt, um dadurch eine Totalfixation $\%$ erhalten.

Zunächst w urde der M. omohyoideus von der Ursprungs- bis zu der Ansatzstelle total exstirpiert und in der üblichen Weise in $10 \%$ iger Formalinlösung weiter fixiert. Dann nahm man aus der $Z_{w i s c h e n-}$ sehne ein Stückchen, aus dem Venter cranialis und aus Venter caudalis 
je vier, im ganzen also neun Gewebsstückchen heraus, entwässerte sie mit allmählich verstärktem A thylalkohol.

Aus diesen Gewebsstückchen verfertigte man Celloidinschniite, wobei man achtgab, daß die Muskelfasern möglichst vollkommen quergeschnitten werden, und einen Teil davon färbte man mit Eisenhämatoxylin, den anderen Teil mit WEIGERTschem Resorzinfuchsin. Wenn man die Dicke der verschiedenen Muskelfasern einwandfrei bestimmen will, so empfiehlt es sich, Flächenmessung an den Querschnitten vorzunehmen. Die Flächenmessung an den Querschnitten haben wir folgendermaßen ausgeführt : Man zeichnet 100 Querschnitte der Muskelfasern mit Hilfe eines Zeichenapparates in 800 facher Vergrößerung auf ein Blatt Papier ab, rechnet die Dimension der Bilder durch den Planimeter möglichst genau aus und setzt die so erhaltenen Zahlen in den absoluten Wert um. In den nachstehenden zwei Tabellen geben wir den arithmetischen Mittelwert von der Dimension der Faserquerschnitte an, welcher mit Quadratmikron ausgedrückt ist; hier ist er aber einfachheitshalber mit $\mu^{2} / 100, d . h$. hundertteilig verkleinert bezeichnet.

\section{Untersuchungsergebnisse.}

\section{a) Dicke der Muskelfasern.}

Beim Venter cranialis haben wir die Stückchen von der Ansat\%stelle an Os hyoideum ab als V. cr. 1, V.cr. 2, V. cr. 3 und V. cr. 4 und beim Venter caudalis von der Zwischensehne ab als V. caud. 1, V. caud. 2, V. caud. 3 und V. caud. 4 bezeichnet. Die Resultate sind in der Tabelle 2 zusammengestellt. Über die Zwischensehne werden wir später berichten.

Tabelle 2. Dicke der Muskelfasern.

\begin{tabular}{|c|c|c|c|c|c|c|}
\hline \multirow{2}{*}{ Mnskel } & \multicolumn{2}{|c|}{17 J. 人ิ } & \multicolumn{2}{|c|}{$29 \mathrm{~J} . \quad \hat{~}$} & \multicolumn{2}{|c|}{$69 \mathrm{~J} . \quad \hat{\delta}$} \\
\hline & $\mathrm{M} \pm \mathrm{m}$ & $\mathrm{V}$ & $\mathrm{M} \pm \mathrm{m}$ & $\mathrm{V}$ & $\mathbf{M} \pm \mathrm{m}$ & $\mathrm{V}$ \\
\hline V.cr. & $2.7 \pm 0.10$ & 40 & $7.5 \pm 0.33$ & 43 & $5.0 \pm 0.28$ & 56 \\
\hline V. cr. & $3.2 \pm 0.09$ & 27 & $7.0 \pm 0.25$ & 35 & $5.2 \pm 0.26$ & 50 \\
\hline V.cr. & $2.6 \pm 0.09$ & 30 & $8.6 \pm 0.49$ & 57 & $4.3 \pm 0.22$ & 50 \\
\hline V.cr. & $3.1 \pm 0.05$ & 16 & $13.6 \pm 0.47$ & 39 & $4.3 \pm 0.25$ & 59 \\
\hline V. caud. 1 & $3.3 \pm 0.11$ & 34 & $13.7 \pm 0.46$ & 34 & $5.0 \pm 0.31$ & 62 \\
\hline $\mathrm{V}$ caud. 2 & $3.2 \pm 0.09$ & 27 & $8.2 \pm 0.45$ & 28 & $6.2 \pm 0.36$ & 58 \\
\hline V. caud 3 & $3.6 \pm 0.19$ & 52 & $10.1 \pm 0.50$ & 49 & $6.1 \pm 0.37$ & 61 \\
\hline $\mathrm{V}$, caud. 4 & $3.1 \pm 0.08$ & 21 & $15.9 \pm 0.48$ & 28 & $5.3 \pm 0.29$ & 54 \\
\hline
\end{tabular}


Tabelle 2. Dicke der Muskelfasern (Fortsetzung).

\begin{tabular}{l|r|c|c|c}
\hline \multirow{2}{*}{ Muskel } & \multicolumn{2}{|c|}{$33 \mathrm{~J}}$. & \multicolumn{2}{|c}{$45 \mathrm{~J}}$. \\
\cline { 2 - 5 } & $\mathrm{M} \pm \mathrm{m}$ & $\mathrm{V}$ & $\mathrm{M} \pm \mathrm{m}$ & $\mathrm{V}$ \\
\hline V.cr. 1 & $4.1 \pm 0.22$ & 53 & $7.3 \pm 0.23$ & 45 \\
V.cr. 2 & $3.9 \pm 0.19$ & 49 & $7.3 \pm 0.23$ & 37 \\
V.cr. 3 & $4.1 \pm 0.27$ & 66 & $8.3 \pm 0.23$ & 33 \\
V.cr. 4 & $4.6 \pm 0.26$ & 56 & $8.8 \pm 0.25$ & 25 \\
V. caud. 1 & $6.1 \pm 0.43$ & 71 & $6.5 \pm 0.29$ & 38 \\
V. caud. 2 & $11.9 \pm 0.60$ & 40 & $4.5 \pm 0.37$ & 53 \\
V. caud. 3 & $3.2 \pm 0.10$ & 30 & $8.2 \pm 0.36$ & 42 \\
V. caud. 4 & $6.8 \pm 0.45$ & 66 & $7.5 \pm 0.31$ & 41
\end{tabular}

Wenn man die in der Tabelle 2 stehenden Werte nach der Dicke der Muskelfasern anreiht, sò ergibt sich folgendes.

Tabelle 3.

\begin{tabular}{|c|c|c|c|c|}
\hline & \multicolumn{2}{|c|}{ Venter cranialis } & \multicolumn{2}{|c|}{ Venter caudalis } \\
\hline & $\begin{array}{c}\text { GröBte } \\
\text { Dicke d. Fasern }\end{array}$ & $\begin{array}{c}\text { Geringere } \\
\text { Dicke d. Fasern }\end{array}$ & $\begin{array}{c}\text { Größte } \\
\text { Dicke d. Fasern }\end{array}$ & $\begin{array}{c}\text { Geringere } \\
\text { Dicke d. Fasern }\end{array}$ \\
\hline $17 \mathrm{~J}$. . . & V. cr. 2 & V. cr. 4 & V. caud. 3 & V. caud. 1 \\
\hline $29 \mathrm{~J} . \quad \hat{8}$ & V. cr. 4 & V. cr. 3 & V. caud. 4 & V. caud. 1 \\
\hline $69 \mathrm{~J} . \quad \hat{8}$ & V. cr. 2 & V. cr. 1 & V. caud. 2 & V. caud. 3 \\
\hline $39 \mathrm{~J} . \quad$ 우 & V. cr. 4 & V. cr. 3 & V. caud. 2 & V. caud. 4 \\
\hline $45 \mathrm{~J} . \quad$ क & V. cr. 4 & V. cr. 3 & V. caud. 3 & V. caud. 4 \\
\hline
\end{tabular}

Im Venter cranialis sind die Muskelfasern meist in der Nähe der Zwischensehne am dicksten, im Venter caudalis aber haben wir die größte Dicke in der Nähe der Zwischensehne und im untersten Abschnitt des Muskels. Was den Dickenunterschied zwischen den Muskelfasern des Venter cranialis und denen des V. caudalis betrifft, so sind die letzteren, worauf KOHASHI (1937) aufmerksam gemacht hat, allgemein etwas dicker.

Ein Unterschied der Muskelfaserdicke bei den verschiedenen Geschlechten ist nicht $z u$ erkennen. Das Körpergewicht dagegen verursacht einen auffallenden Unterschied, und zwar sind bei größerem Körpergewicht bzw. bei besserer Ernährung die Muskeltasern dicker.

Der Variationskoeffizient wächst mit zunehmendem Alter. Er ist kleiner bei Jüngeren, und größer bei Alteren. Beim mittleren Alter ist er im allgemeinen mittelgroß. 


\section{b) Gesamtzahl der Fasern im Muskelquerschnitt in jeder Höhe.}

Den ganzen Querschnitt in jeder Höhe haben wir in 250 facher Vergrößerung photographiert und die Zahl der Muskeliasern mit der Nadel stechend festgestellt.

Tabelle 4. Gesamtzahl der Fasern im Muskelquerschnitt.

\begin{tabular}{|c|c|c|c|c|c|}
\hline & $17 \mathrm{~J}$. ô & $29 \mathrm{~J}$. 今 & $69 \mathrm{~J} . \hat{o}$ & $33 \mathrm{~J}$. 우 & $45 \mathrm{~J}$. ? \\
\hline V.cr. & 14839 & 12643 & 6165 & 8993 & 14138 \\
\hline v.cr. 2 & 13715 & 17242 & 7323 & 5664 & 22714 \\
\hline V.cr. 3 & 12285 & $145: 7$ & 6637 & 6519 & 14499 \\
\hline V.cr. 4 & 5000 & 2404 & 4678 & 3656 & 2889 \\
\hline V.caud. 1 & 9558 & 69.53 & 3832 & 8487 & 6876 \\
\hline V. caud. 2 & 11347 & 14650 & 3888 & 9878 & 23250 \\
\hline V. caud. 3 & 12015 & 21060 & 9624 & 9132 & 23054 \\
\hline V. caud. 4 & 10936 & 12974 & 9854 & 7109 & 22804 \\
\hline
\end{tabular}

Die Muskelfasern sind in der Nähe der Zwischensehne, des Os hyoideum und des oberen Randes der Scapula in geringerer Zahl. Die Zahl der Fasern nimmt an dem Übergangsteil zur Sehne bedeutend ab. In Muskelbauch befinden sich aber zahlreichere Muskelfasern.

\section{c) Mikroskopischer Befund an der Mittelsehne.}

Es gibt Fälle, wo V. cranialis und caudalis durch die Mittelsehne voneinander vollkommen getrennt sind, und auch Fälle, wo sie nur unvollkommen getrennt sind. Es ist noch zu erwähnen, daß bei $69 \mathrm{~J}$. $\hat{\delta} \operatorname{der} Z_{W}$ ischensehnenanteil zum größten Teil aus dem Muskelgewebe lesteht, und daß die Sehne seitlich nur ein wenig zu sehen ist. Die Fortsetzung der Sehne ist noch in V.cr. 4, V.cr. 3 und V.caud. 2 vorhanden.

\section{Zusammenfassung.}

1. Die Faserdicke des M. omohyoideus des Menschen zeigt nur wenigen Unterschied zwischen den beiden Geschlechten, aber großen Unterschied je nach dem Körpergewicht und Alter. Wenn man z. B. den obersten Teil des Venter cranialis aus einen 29 jährigen Mann von Körpergewicht $60.1 \mathrm{~kg}$ mit dem aus einer 33 jährigen Frau von $48.2 \mathrm{~kg}$ vergleicht, so beträgt die Faserdicke $\left(\mu^{2} / 100\right)$ beim ersteren 7.5 und beim letzteren 4.1. Betreffs des Alters zeigt der oberste Teil des Venter cranialis bei einem 17.jährigen Mann 2.7, bei einem 29 jährigen Mann 7.5, bei einem 69 jährigen Mann 5.0, bei einer 33 jährigen Frau 
4.1 und bei einer 45 jährigen Frau 7.3. Beim ersten Mann sind die Mruskelfasern atßerordentlich dünn, weil er wegen Lungentuberkulose. sehr abgemagert war. Auch an den anderen Abschnitten des Muskels sind die Dickenverhältnisse der Muskelfasern im wesentlichen die gleichen.

2. Der Variationskoeffizient der Dicke der Muskelfasern ist in der Regel kleiner bei Jüngeren, dagen größer bei zunehmendem Alter und mittelgro $B$ beim mittleren Alter. Z. B. ist er an dem Querschnitt des obersten Teil des Venter cranialis beim 17 jährigen Mann 40, beim 29jährigen Mann 43, beim 69 jährigen Mann 56, bei der 33 jahrigen Frau 53 und bei der 45 jährigen Frau 45. Die Verhältnisse des Variationskoeffizienis an anderen Abschnitten des Muskels sind auch im wesentliehen die gleichen.

3. Der Venter cranialis und caudalis sind durch die $Z_{w}$ ischensehne bald vollkommen, bald unvollkommen voneinander getrennt.

4. Die dicksten Muskelfasern befinden sich in der Mehrzahl der Fälle im Venter cranialis in der $N$ ähe der $Z_{w}$ ischensehne und im Venter caudalis in der Nähe der $Z_{w}$ ischensehne sowie des Ansatzes an der Scapula.

5. Die Gesamtzahl der Muskelfasern ist in der Mitte des Muskelbauches (des Venter cranialis und des Venter caudalis) am größten.

\section{內 容自抄}

局甲舌骨筋は小筋ではあるが，中間腱によつて、上下の 2 腹に分れ居り，

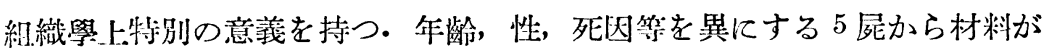
搝られた・よく檢すると，筋は中間腱により完全に分れて居るてとと不完 全に分れて居るてととあり，筇線維の最も太んのは多くの場合上腹では中 注腱に近いとてろで，下腹では中間腱に近いとてろと危用骨への附着部に 近いとてろである・筋線維の總數は筋腹の中央に最も多い. 筋線維の太さ の變異係數は通例若年に小で，高年に大である・笳線維の太さは性による 倠が少く, 體重と年齢による装が多ん.

\section{Literaturverzeichnis.}

Kohashi, Y. : Histologische Untersuchungen der verschiedenen Skeletmuskeln beim Menschen. I. Untersuchungen beim Erwachsenen. Okajimas Fol. anat. jap. 15 (1937). 